\title{
Heat Transfer Prediction for Methane in Regenerative Cooling Channels with Neural Networks
}

\author{
G. Waxenegger-Wilfing, $\stackrel{*}{*}$ K. Dresia, $\stackrel{ \pm}{ \pm}$ J. C. Deeken,,$\stackrel{ \pm}{ \pm}$ and M. Oschwald $\stackrel{\S}{\$}$ \\ DLR, German Aerospace Center, 74239 Hardthausen, Germany
}

\begin{abstract}
https://doi.org/10.2514/1.T5865
Methane is considered a good choice as a propellant for future reusable launch systems. However, the heat transfer prediction for supercritical methane flowing in the cooling channels of a regeneratively cooled combustion chamber is challenging. Because accurate heat transfer predictions are essential to design reliable and efficient cooling systems, heat transfer modeling is a fundamental issue to address. Advanced computational fluid dynamics (CFD) calculations achieve sufficient accuracy, but the associated computational cost prevents an efficient integration in optimization loops. Surrogate models based on artificial neural networks (ANNs) offer a great speed advantage. It is shown that an ANN, trained on data extracted from samples of CFD simulations, is able to predict the maximum wall temperature along straight rocket engine cooling channels using methane with convincing precision. The combination of the ANN model with simple relations for pressure drop and enthalpy rise results in a complete reduced-order model, which can be used for numerically efficient design space exploration and optimization.
\end{abstract}

\section{Nomenclature}

$A=$ channel area, $\mathrm{mm}^{2}$

$A R=$ aspect ratio

$b=$ channel width, $\mathrm{mm}$

$D_{h}=$ hydraulic diameter, $\mathrm{mm}$

$d=$ wall thickness, $\mathrm{mm}$

$f=$ friction factor

$G=$ mass-flow density, $\mathrm{kg} \cdot \mathrm{s}^{-1} \cdot \mathrm{m}^{-2}$

$h=$ channel height, $\mathrm{mm}$

$h \quad=$ specific enthalpy, $\mathrm{J} \cdot \mathrm{kg}^{-1}$

$k=$ number of categories-

$l=$ channel length, $\mathrm{mm}$

$\dot{m}=$ mass-flow rate, $\mathrm{kg} \cdot \mathrm{s}^{-1}$

$p=$ pressure, $\mathrm{Pa}$

$\dot{Q}=$ heat flow rate, $\mathrm{W}$

$\dot{q}=$ heat flux, $\mathrm{W} \cdot \mathrm{m}^{-2}$

$R e=$ Reynolds number

$r=$ wall roughness, $\mu \mathrm{m}$

$T=$ temperature, $\mathrm{K}$

$v=$ flow velocity, $\mathrm{m} \cdot \mathrm{s}^{-1}$

$y^{+}=$dimensionless wall distance

$z=$ flow length, $\mathrm{mm}$

$\alpha=$ regularization parameter

$\epsilon=$ learning rate

$\theta=$ network parameter

$\rho \quad=$ density, $\mathrm{kg} \cdot \mathrm{m}^{-3}$

$\phi=$ activation function

$\Omega=$ weight penalty term

Subscripts

$b \quad=$ bulk

Received 26 July 2019; revision received 20 September 2019; accepted for publication 18 November 2019; published online Open Access 10 January 2020. Copyright $\odot 2019$ by DLR (German Aerospace Center). Published by the American Institute of Aeronautics and Astronautics, Inc., with permission. All requests for copying and permission to reprint should be submitted to CCC at www.copyright.com; employ the eISSN 1533-6808 to initiate your request. See also AIAA Rights and Permissions www.aiaa.org/randp.

*Research Scientist, Institute of Space Propulsion, Rocket Engine Department; Guenther. Waxenegger@ dlr.de.

${ }^{\dagger} \mathrm{Ph}$.D. Student, Institute of Space Propulsion, Rocket Engine Department; Kai. Dresia@dlr.de.

${ }^{\ddagger}$ Group Leader, Institute of Space Propulsion, Rocket Engine Department; Jan. Deeken@dlr.de.

${ }^{\S}$ Department Head, Institute of Space Propulsion, Rocket Engine Department; Michael. Oschwald@dlr.de.

$\begin{array}{ll}i & =\text { total number of trainable weights } \\ \text { in } & =\text { inlet } \\ j & =\text { summation index } \\ n & =\text { dimension of input vector } \\ \text { out } & =\text { outlet } \\ \text { stat } & =\text { static } \\ \text { tot } & =\text { total } \\ w & =\text { wall }\end{array}$

\section{Introduction}

A LTHOUGH most liquid rocket engines that have flown until now used liquid oxygen (LOX)/hydrogen $\left(\mathrm{LH}_{2}\right)$, liquid oxygen/ kerosene (rocket propellant-1 or RP-1), or a hypergolic propellant combination like nitrogen tetroxide (NTO)/monomethylhydrazine (MMH) [1,2], several countries have started to develop engines that use methane $\left(\mathrm{CH}_{4}\right)$ as fuel and oxygen as oxidizer in recent years. On the one hand, there are development projects by national agencies such as the Japan Aerospace Exploration Agency [3], the ESA [4], NASA [5] , and DLR, German Aerospace Institute [6] ; on the other hand, various rocket engines are developed by private companies such as BE4 by Blue Origin and Raptor by SpaceX. Oxygen/hydrogen offers the highest specific impulse, but the low density of hydrogen leads to large rocket stages. In addition, the low boiling temperature of hydrogen at $20 \mathrm{~K}$ makes the handling very difficult and increases operating costs. Kerosene is much denser than hydrogen and easier to handle. Disadvantages are a lower specific impulse and that kerosene may coke and form deposits, which is problematic in terms of engine reuse. The main drawback of nitrogen tetroxide/monomethylhydrazine is its extreme toxicity. The propellant combination of oxygen/methane has many favorable characteristics; e.g., methane is six times as dense as hydrogen, is easier to handle, and has preferable coking temperature limits [7] and low toxicity. Furthermore, oxygen/methane offers a slightly higher specific impulse than oxygen/kerosene []].

Despite the mentioned advantages, the prediction of heat transfer for methane flowing in the cooling channels of a regeneratively cooled combustion chamber has proven challenging [9], but it is needed for an efficient cooling system design. Regenerative cooling performance is especially important for engines, which are reusable or use an expander (bleed) power cycle, where the energy absorbed in the cooling channels drives the turbopumps $[10,11]$. The main difficulty for the heat transfer prediction of methane is that it usually enters the cooling channels at supercritical pressure but subcritical temperature. It is then heated up in the cooling channels and, most times, crosses the Widom line [12] close to the critical point. The Widom line extends the liquid-vaporcoexistence line into the supercritical region by using the thermodynamic locus of the specific heat maxima [13]. Strong changes in fluid properties at the Widom line introduce various physical phenomena 
(e.g., heat transfer deterioration $[14,15])$, which influence the heat transfer. In contrast to that, hydrogen usually enters the cooling system, which is already in a gaslike state, with pressures and temperatures far above the critical values [16].

Several methods exist to study the regenerative cooling of liquid rocket engines. A simple approach is to use semiempirical one-dimensional correlations to estimate the local heat transfer coefficient $[17,18]$. By using an energy balance for each combustion chamber wall section, the wall temperatures can be estimated. The maximum wall temperature, which occurs at the hot-gas side, is an especially critical parameter because it determines the fatigue life of the chamber [19]. The advantage of such simple relations is the negligible computation time. However, one-dimensional relations are not able to capture all relevant effects that occur in asymmetrically heated channels, like thermal stratification [20] or the influence of turbulence and wall roughness. Correction factors and quasi-two-dimensional models have been developed [21], but only

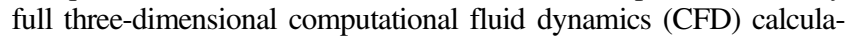
tions achieve convincing accuracy. Many papers have been published on CFD simulations for supercritical methane flowing in rocket engine cooling channels [22-25], and CFD results were compared with experimental data $[26,27]$. The main disadvantage of three-dimensional CFD simulations is that they are not suitable for design optimization, design space exploration, and sensitivity analysis due to their large calculation effort.

By constructing surrogate models using samples of the computationally expensive calculation, one can alleviate this burden. However, it is crucial that the surrogate model mimics the behavior of the simulation model as closely as possible and generalizes well to unsampled locations while being computationally cheap to evaluate. Artificial neural networks (ANNs) are known to be universal function approximators [28] and have been successfully applied as surrogate models in a number of domains $[29,30]$. These models have been applied to the heat transfer prediction of supercritical fluids too [31-33]. The possibility to use ANNs with multiple hidden layers allows the generation of surrogate models, even for high-dimensional problems, given a suitable number of samples. In this paper, for the first time, an ANN is trained with data extracted from samples of CFD simulations for heat transfer prediction of supercritical methane. The rest of the paper is organized as follows: Sec. II describes the basics of machine learning (ML) and the theory of ANNs. A procedure to generate suitable training data by CFD calculations is presented in Sec. III. Section IV discusses the proposed ANN and reports the results. Section $\mathrm{V}$ shows how the ANN can be used

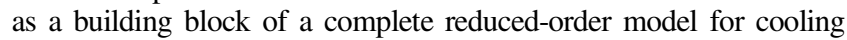
channel flows, and Sec. VI provides concluding remarks. A good deal of the material presented in the paper can also be found in the master's thesis of Dresia, which was supervised by Waxenegger-Wilfing [34].

\section{Artificial Neural Networks}

ANNs are models that belong to the field of ML. To understand ANNs well, a basic understanding of the principles of ML is needed. The following section briefly elaborates on the basic theory. A comprehensive presentation can be found in the book of Goodfellow et al. [35].

\section{A. Machine Learning Basics}

The field of ML studies algorithms that use datasets to change parts of a mathematical model in order to solve a certain task instead of using fixed predefined rules. The mathematical model is often a function, which maps input data to output data, and the algorithm has to learn the adjustable parameters of this function in such a way that the mapping has the desired properties. In other words, ML is primarily concerned with the problem of finding and adjusting functions that usually have a large number of parameters. ML algorithms can be divided into supervised and unsupervised. In supervised learning, the training dataset contains both the inputs and the desired outputs, and the mathematical model can (among other things) be used for classification or regression. In a classification task, the model is asked to identify to which set of categories $k$ a specific input belongs. Assuming that each example of the input data is represented as a feature vector $\boldsymbol{x} \in \mathbb{R}^{n}$, the learning algorithm is asked to produce a suitable function $f: \mathbb{R}^{n} \rightarrow\{1, \ldots, k\}$ with a discrete target output. A well-known example of a classification task is object recognition in images. In a regression task (e.g., with a single explanatory variable), the goal is to predict a numerical value given some input. To solve this task, the learning algorithm is asked to output a function $f: \mathbb{R}^{n} \rightarrow \mathbb{R}$. Unsupervised learning algorithms receive datasets without target outputs and learn useful properties of the structure of these datasets.

The central challenge in ML is that the model must perform well on new, previously unseen input data. The capability to perform well on those inputs is called generalization. Generalization is also central to understand the relationship between mathematical optimization and ML. Although optimization algorithms can be used to minimize some error measure on the training set, ML tries to reduce the generalization error, which is also called the test error. During training, one must prevent two central issues. Underfitting occurs when the model is not able to obtain a sufficiently low error on the training data. Overfitting occurs when the gap between the training error and the test error is too large; thus, the model is not able to generalize. The ability of a model to fit a wide variety of functions is called the model's capacity. Models with low capacity may have problems fitting the training data. Models with high capacity can solve complex tasks; but, when their capacity is higher than needed, they may overfit by memorizing properties of the training data that do not work well on the previously unseen test data. ML achieves good results when the capacity of the model is appropriate for the true complexity of the relevant task and the amount of training data. However, for practical applications, it is nearly impossible to guess the model with an appropriate capacity. Furthermore, models with higher capacity in combination with proper methods to prevent overfitting often work better than less complex models. Modifications of a learning algorithm that are intended to reduce its generalization error, possibly by an increase in training error, are known as regularization. Instead of reducing the capacity of the model, one can, for example, change the learning algorithm to express the preference of one function over another.

Most ML models and algorithms have hyperparameters that are not adapted by the learning algorithm but can be used to control the outcome, for example, by changing the capacity of a model. Optimal values of hyperparameters and estimates for the generalization error are found by splitting the available data into three disjoint subsets. The training set is used to adapt the trainable parameters by the learning algorithm. The second dataset, which is the validation set, exists to estimate the generalization error during or after training, allowing for hyperparameter tuning with the goal to find a good balance between performance and avoidance of overfitting. However, the estimate of the generalization error of the final model will be biased because the validation data were used to select the model. Thus, a third dataset, which is the test set, is used to estimate the real generalization error.

\section{B. Theory of Artificial Neural Networks}

One successful family of models used for ML is that of ANNs. ANNs are inspired by the functionality of biological brains, which are made of a huge number of biological neurons that work together to control the behavior of animals and humans. A collection of connected units, called artificial neurons, forms the basis of an ANN. Furthermore, artificial neurons loosely model biological neurons and are usually represented by nonlinear functions acting on the weighted sum of its input signals. Let $\boldsymbol{i n}=\left(i n_{1}, i n_{2}, \ldots, i n_{n}\right)$ denote an input vector, $\boldsymbol{w}=\left(w_{1}, w_{2}, \ldots, w_{n}\right)$ a weight vector (where $n$ is the input dimension), $b$ a bias term, and $\phi$ an activation function; then, the output (out) of a single artificial neuron can be written as

$$
\text { out }=\phi\left(\sum_{j=1}^{n} w_{j} i n_{j}+b\right)
$$

The bias term $b$ can be used to shift the activation function $\phi$. A rectified linear unit $(\operatorname{ReLU})$, where $\phi(x)=\max \{0, x\}$, is the most common activation function in modern ANNs. Trainable parameters are usually the weights and biases of the neurons. Mostly, the connectivity architecture of such ANNs is layered with an input layer, 
multiple hidden layers, and an output layer. ANNs are called feedforward networks when no feedback connections are present. One can prove that a feedforward network with a single hidden layer can approximate any reasonable function if the hidden layer has enough neurons. Nevertheless, using multiple hidden layers adds exponentially more expressive power. Among other things, each layer can be used to extract increasingly abstract features and hence more suitable representations of the input data. An ANN with more than one hidden layer is called a deep ANN. Such a deep ANN can discover a suitable hierarchy of representations during training and, as a result, learn and generalize better. During training or learning, the algorithm requires a measure for the quality of its prediction to adjust the parameters of the model. In regression problems, a typical choice for the cost function is the mean squared error between the predicted values and ground truth:

$$
J(\boldsymbol{\theta})=\frac{1}{2 m} \sum_{\boldsymbol{x}}(y(\boldsymbol{x})-f(\boldsymbol{x}, \boldsymbol{\theta}))^{2}
$$

where $\boldsymbol{x}$ and $y(\boldsymbol{x})$ are the input vector and the ground truth, respectively, of a training data point; $m$ is the total number of training data points; and $f(\boldsymbol{x}, \boldsymbol{\theta})$ is the predicted output of the model according to its parameters $\boldsymbol{\theta}$. For ANNs, the model parameters $\boldsymbol{\theta}$ are given by all weights and biases associated to the neurons. Training corresponds to finding optimal parameters $\boldsymbol{\theta}$ such that $J(\boldsymbol{\theta})$ is minimal. Often, one adds an extra term for regularization:

$$
\begin{aligned}
\tilde{J}(\boldsymbol{\theta}) & =J(\boldsymbol{\theta})+\alpha \Omega(\boldsymbol{\theta}) \quad \text { with } \\
\Omega(\boldsymbol{\theta}) & =\frac{1}{2}\|\boldsymbol{w}\|_{2}^{2}=\frac{1}{2}\left(w_{1}^{2}+w_{2}^{2}+\ldots+w_{i}^{2}\right)
\end{aligned}
$$

where $\boldsymbol{w}$ denote the weights of the ANN; $i$ is the total number of trainable weights; and $\alpha$ is an additional hyperparameter, which controls the amount of regularization. The extra term penalizes larger network weights. The procedure is known as weight decay or L2 regularization. Because of the nonlinearity of ANNs, $J(\boldsymbol{\theta})[\operatorname{or} \tilde{J}(\boldsymbol{\theta})]$ is a nonconvex function. One can still use gradient-based optimizers, but there is no global convergence guarantee. Nevertheless, the training algorithms of ANNs are mostly based on using the gradient to descend the cost function to lower values. After initializing all trainable parameters (for example, by small random numbers), the gradient of the cost function is used to update the parameters by

$$
\boldsymbol{\theta}^{\prime}=\boldsymbol{\theta}-\epsilon \nabla J
$$

where $\epsilon$ is a small parameter called the learning rate that ensures that the change in $\boldsymbol{\theta}$ is small. The gradient $\nabla J$ of the cost function with respect to $\boldsymbol{\theta}$ can efficiently be computed with the backpropagation algorithm. For large training datasets, gradient computation can still be very time consuming. It turns out that the efficiency can be improved by calculating the gradient on small randomized subsets of the training set, called minibatches, and applying updates to the parameters more often. This procedure is called stochastic gradient descent. Finally, one pass of the full training set is called an epoch.

The use of ANNs for surrogate modeling has advantages and disadvantages. A big advantage is that ANNs can capture the behavior of complicated functions because they can scale to large datasets and generalize nonlocally [35]. Especially if a deep network can extract the underlying factors, ANNs are well suited even for highdimensional problems. The biggest disadvantage is that ANNs mostly act as black boxes. The field of explainable artificial intelligence exists to make models like ANNs more explainable and interpretable, but it is still in its infancy.

\section{CFD-Based Data Generation}

For the generation of training and test datasets, CFD calculations of supercritical methane flowing inside of straight cooling channel segments are performed. As mentioned in the Introduction (Sec. I), many studies have been performed to derive suitable CFD setups, which can reproduce all essential effects influencing the heat transfer. The focus of this paper is to show the feasibility of ANNs to tackle the challenge of numerically efficient heat transfer predictions under the assumption that precise CFD solvers are available for the corresponding problem setting.

\section{A. CFD Models}

The CFD models are generated with standard ANSYS CFX 18.0. CFX solves the Navier-Stokes equations including viscous terms using an element-based finite volume approach for the space domain and a second-order backward Euler scheme for time discretization [36]. The channel flow is modeled as compressible and steady, whereas buoyancy and gravitational forces are neglected. Turbulence is predicted using the two-equation shear stress transport model, which combines the $k-\omega$ turbulence model for the inner region of the boundary layer with the $k-\epsilon$ turbulence model for the free shear flow. The geometry and boundary conditions of the cooling channel model are shown in Figs. 1 and 2. Because of symmetry reasons, it is sufficient to model one-half of the channel. Note that $h$ and $b$ denote the channel height and width, whereas $d$ is used for the chamber wall thickness in Fig. 1. To restrict the independent variables, a fin thickness of $1 \mathrm{~mm}$ is assumed for all simulations. In the streamwise direction, no heat flux $\left(\dot{q}=0 \mathrm{~W} \cdot \mathrm{m}^{-2}\right)$ is applied for the first $80 \mathrm{~mm}$ of the channel to obtain a fully developed flow and velocity boundary layer. Also, $l$ denotes the channel length and is set to $250 \mathrm{~mm}$ for a cross section smaller than or equal to $5 \mathrm{~mm}^{2}$, whereas it is increased for channels with a larger cross section to allow the thermal boundary layer to grow further. The channel surface is modeled as a rough wall with different values for the surface roughness and a no-slip condition. A mass-flow boundary condition and

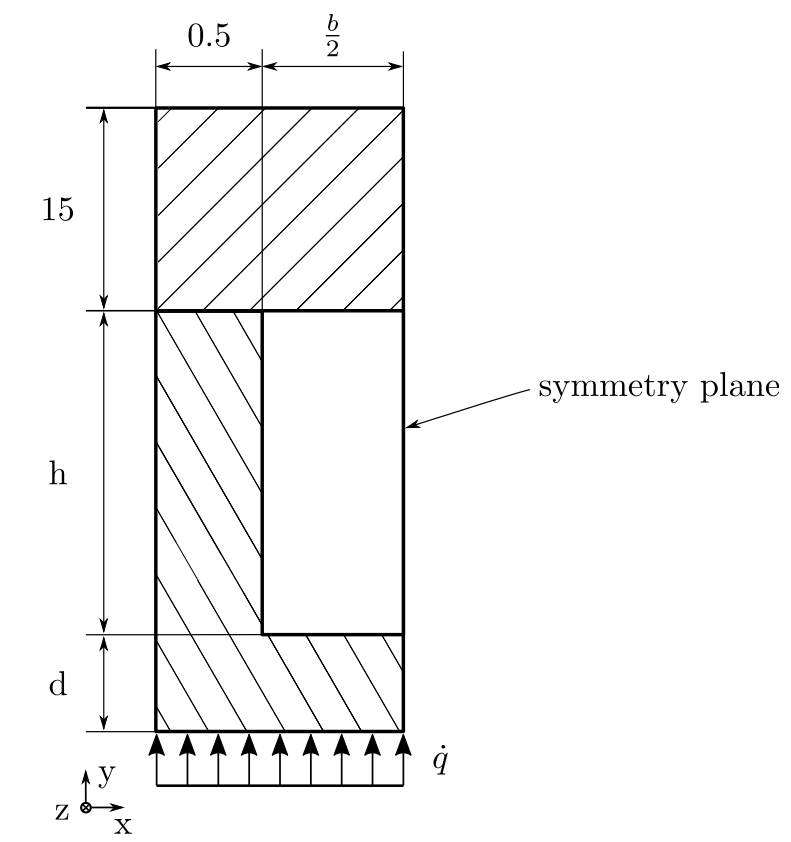

Fig. 1 Geometry and boundary conditions of the cooling channel (not to scale).

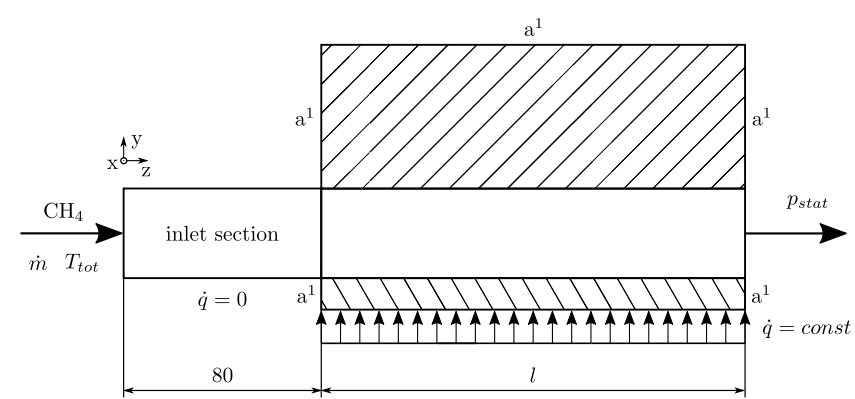

Fig. 2 Computational domain with boundary conditions (not to scale) $-{ }^{1}$ walls denoted with "a" are adiabatic. 
the coolant total temperature are imposed at the fluid inlet. Furthermore, the static pressure is fixed at the domain outlet and a symmetric flow boundary condition assures no mass or energy fluxes across the symmetry plane. For the solid domain, all faces (except the hot-gas wall) are modeled as adiabatic walls. The thermodynamic properties of supercritical methane are evaluated with data from the well-known National Institute of Standards and Technology (NIST) database [37], which provides data up to $625 \mathrm{~K}$. For higher temperatures, an ideal gas behavior is assumed. The solid domain uses two different material models. The combustion chamber and solid fins are made of a $\mathrm{CuCrZr}$ alloy, which in this case is $99.25 \%$ copper $(\mathrm{Cu}), 0.62 \%$ chrome $(\mathrm{Cr})$, and $0.1 \%$ zirconium $(\mathrm{Zr})$. For the material properties of the alloy, the reader is referred to the work of Oschwald et al. [38]. The galvanic layer is assumed to be made of copper. The fluidstructure interaction is not considered. To reduce the influence of axial heat transfer, the thermal conductivity in the streamwise direction is set to zero for both materials.

The following parameters are varied for data generation: mass-flow density $G$, heat flux $\dot{q}$, outlet pressure $p_{\text {stat,out }}$, inlet temperature $T_{\text {stat,in, }}$, surface roughness $r$, channel area $A$, aspect ratio $A R$, and inner wall thickness $d$. Their upper and lower bounds are chosen so that the data cover the geometrical dimensions and operation conditions of both upper-stage and first-stage liquid rocket engines with moderate chamber pressure. The outlet pressure ranges between 50 and 150 bar, which means that the fluid pressure is always above the critical pressure of methane; consequently, no boiling or phase change occurs. The fluid inlet temperature varies from 120 to $400 \mathrm{~K}$. Hence, there are simulations where the coolant temperature crosses the Widom line and a transition from a liquidlike to a gaslike state takes place. Furthermore, both outlet pressures and inlet temperatures are clustered more narrowly around the critical point to ensure that these critical cases are well represented. To model both smooth and rougher walls, sand-grain roughnesses between 0.2 and $15 \mu \mathrm{m}$ are considered. The channel area varies from 1 to $10 \mathrm{~mm}^{2}$, and different channel aspect ratios (1.0 to 9.2) are simulated because of their impact on heat transfer and maximum wall temperature. For the channel with a cross section of $1 \mathrm{~mm}^{2}$, only an aspect ratio of 1.0 is used to take the manufacturing restriction into account. The inner chamber wall thickness varies from 0.8 to $1.2 \mathrm{~mm}$, which significantly influences the hot-gas wall temperature. Generally, higher mass-flow densities are considered for high heat fluxes and smoother walls because they result in reasonable wall temperatures and pressure losses.

For both solid and fluid domains, hexahedral mesh elements are generated with ANSYS ICEM. The first element in the boundary layer has a thickness of $0.1 \mu \mathrm{m}$ to satisfy a value of $y+<1$. The grid resolution is $100 \mu \mathrm{m}$ in the streamwise direction and $35 \mu \mathrm{m}$ perpendicular to it for the fluid domain. For grid independence, a finer mesh with twice as many elements was analyzed for certain test cases. Because the resulting wall temperatures only change by $2 \%$, the coarser mesh shown Fig. 3 is used. A converged solution has to fulfill three criteria: All root-mean-square residuals must be below $1 \times 10^{-5}$, the conservation equations are well satisfied (solution imbalances below 1\%), and quantities of interest (such as pressure drop or maximum wall temperature) do not change significantly between two iterations. In total, approximately 20,000 CFD simulations of straight cooling channel segments are performed.

\section{B. CFD Results}

ML techniques can only cover effects if they are already present in the training data. Important phenomena, which affect flows in asymmetrically heated cooling channels and the associated heat transfer, are thermal stratification and heat transfer deterioration. Both effects can be observed in the CFD results; e.g., Fig. 4 shows the maximum wall temperature and mean bulk temperature along the axial direction of a simulated straight cooling channel for different constant wall heat fluxes. The wall temperature distribution exhibits a peak for higher heat fluxes as a consequence of heat transfer deterioration, whereas the bulk temperature increases nearly linearly. The influence of the surface roughness is also modeled correctly. Higher roughness levels enlarge the production of turbulence in the boundary layer.

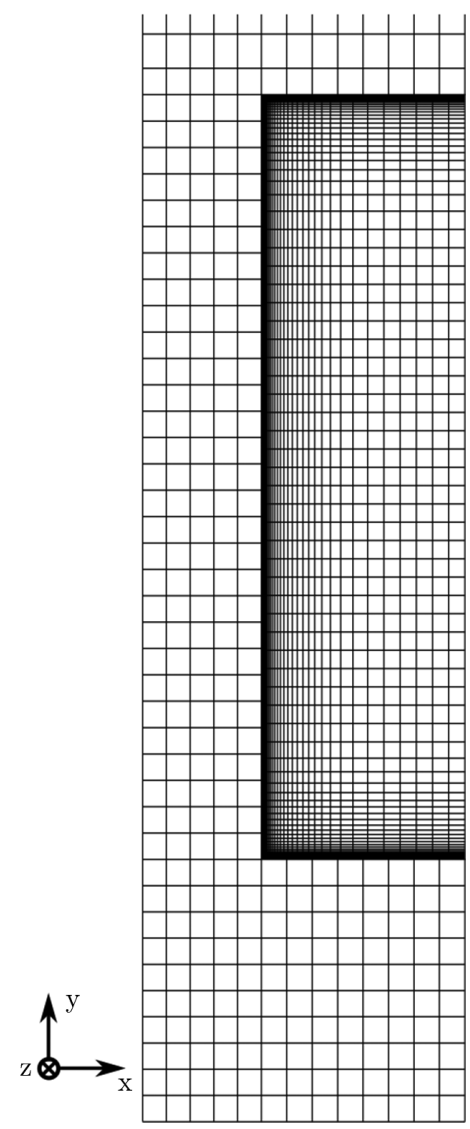

Fig. 3 Computational grid.

Thus, wall temperatures are decreased, but the pressure loss is increased. These implications coincide with the CFD results. Overall, it can be concluded that the relevant consequences of thermal stratification, heat transfer deterioration, and surface roughness are represented in the generated data. However, the proof that standard ANSYS CFX is able to correctly predict the heat transfer for all flow regimes and cooling channel geometries is still missing and the subject of current research.

\section{Data Reduction}

Only a reduced amount of the CFD results is used for training the ANN. First, only the values of bulk properties are used for the fluid description. Bulk properties are calculated as mass-flow-averaged quantities across the channel cross section. Although most information contained in the two-dimensional distribution of fluid quantities is lost, it is hoped that the impact will be reflected in the correlations of the bulk variables. Second, at each cross section, the temperature distribution of the solid part is reduced to the values of the mean wall temperatures and the maximum wall temperature at the hot-gas side. Nevertheless, it would be interesting to check how far the accuracy of a data-driven model can be increased by using a more complete description of the fluid and solid states. Third, these variables are only evaluated every $2 \mathrm{~mm}$ in the streamwise direction and saved in a tablelike file structure. Each data point is extended by the associated geometric information, such as cross-section area, aspect ratio, and flow length, as well as boundary conditions like heat flux and surface roughness. The flow length is used to include boundary-layer effects on the heat transfer.

After data generation, it is always recommended to study the content and distribution of the data. First, a correlation matrix can be used to visualize the correlations between multiple variables. Figure 5 shows the correlation matrix calculated with the generated data for certain variables, where each entry visualizes the value of the corresponding Pearson correlation coefficient. It is important to note that the Pearson correlation only describes the strength of linear relationships and does not imply causation. For example, the correlation coefficient between 


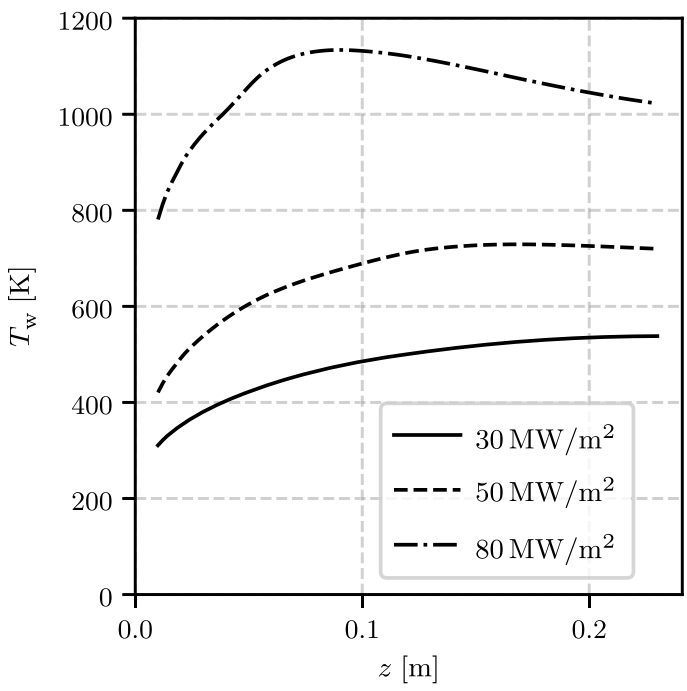

a) Maximum wall temperature $T_{\mathrm{w}}$

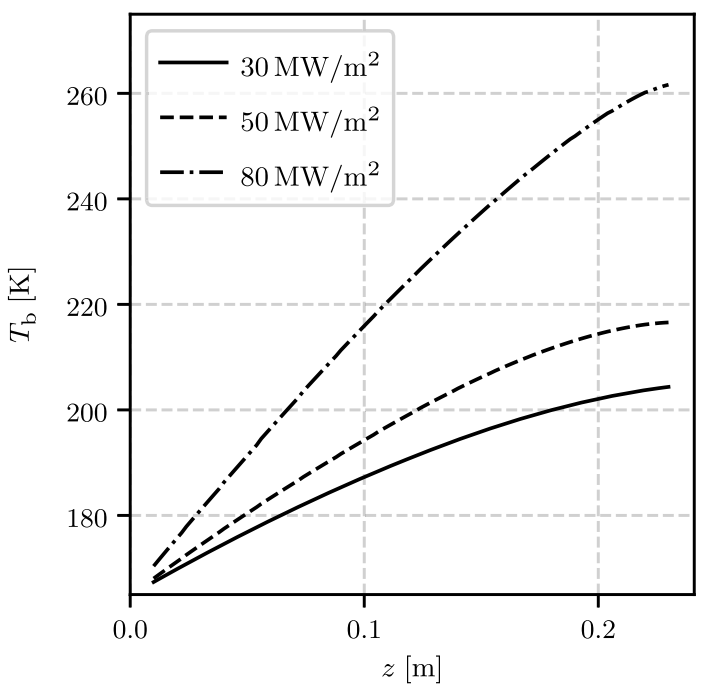

b) Bulk temperature $T_{\mathrm{b}}$

Fig. 4 Wall temperature and bulk temperature for different heat fluxes.

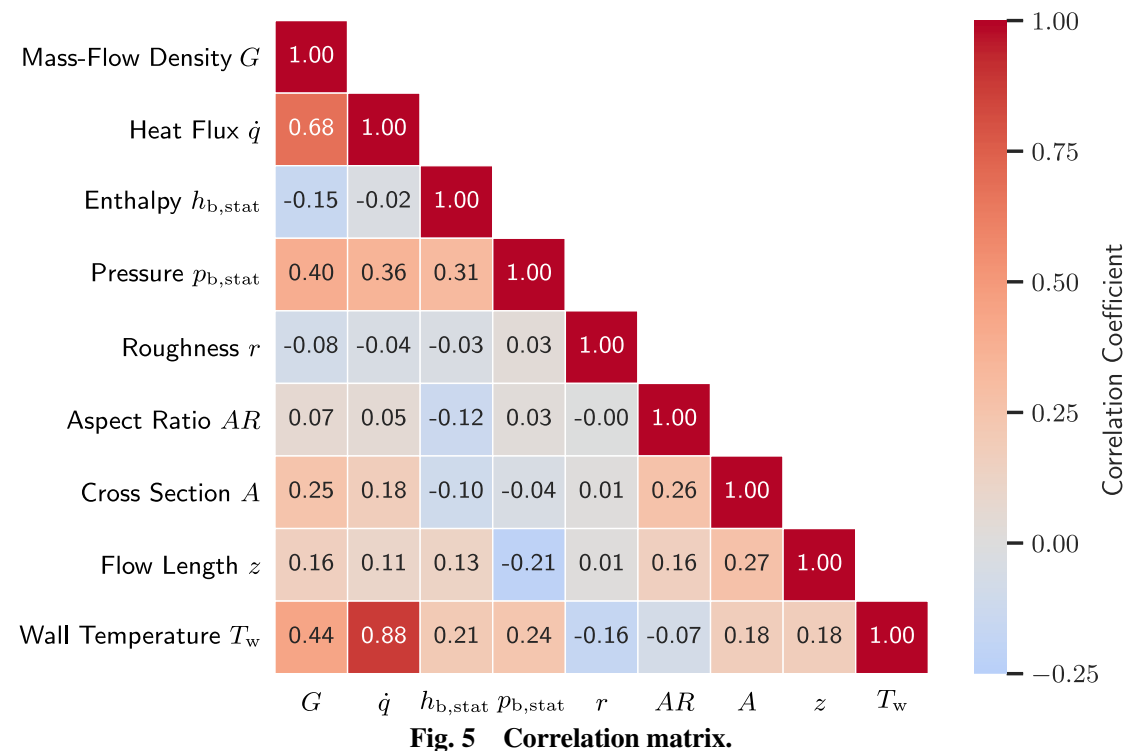

the wall temperature and heat flux is 0.88 and indicates a strong positive relationship; whereas the correlation between the wall temperature and surface roughness is negative, as expected. One can see that physically reasonable relations are still represented in the reduced data. Second, the data are not uniformly distributed and there are regions where the data are very sparse or where no data points are present at all. Figure 6 exemplarily shows the distribution with respect to enthalpy and pressure. As a result of the data generation process with its manually chosen boundary conditions, there are regions with higher and lower data

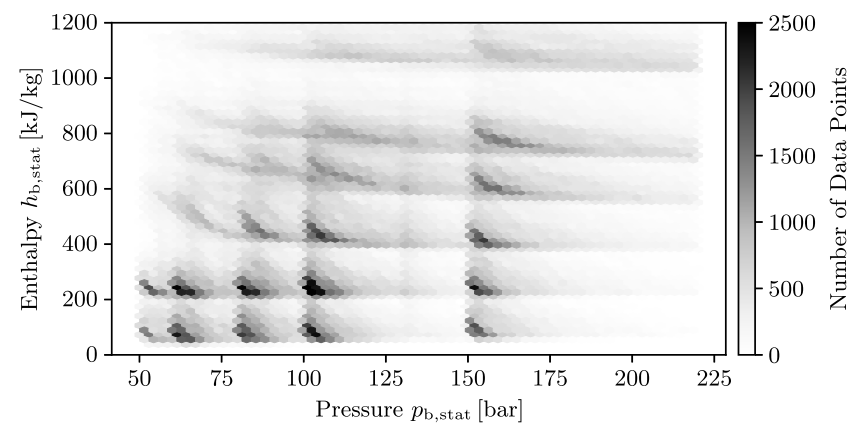

Fig. 6 Data distribution. density. A so-called covariate shift refers to a situation where the distribution of input variables is different in the data available for training and the data one expects to use as input in the future [39]. This needs to be taken into account because the ANN should also produce good predictions there.

\section{Artificial Neural Network for Wall Temperature Prediction}

An important problem is the prediction of the maximum temperature for each section of the combustion chamber wall given a certain cooling channel design and suitable boundary conditions. The maximum temperature is a critical parameter because it directly determines the fatigue life of the chamber, and is therefore a crucial constraint for design considerations [19]. The main driver for the temperature is the heat transfer from the cooling channel to the coolant. Hence, the prediction can only be successful if the implicit heat transfer modeling takes the underlying mechanisms correctly into account. Put differently, this means that an accurate wall temperature prediction implies a proper reduced-order modeling of the relevant heat transfer.

\section{A. Network Architecture and Hyperparameter Optimization}

A fully connected, feedforward network is proposed for the wall temperature prediction. The term fully connected means that every 


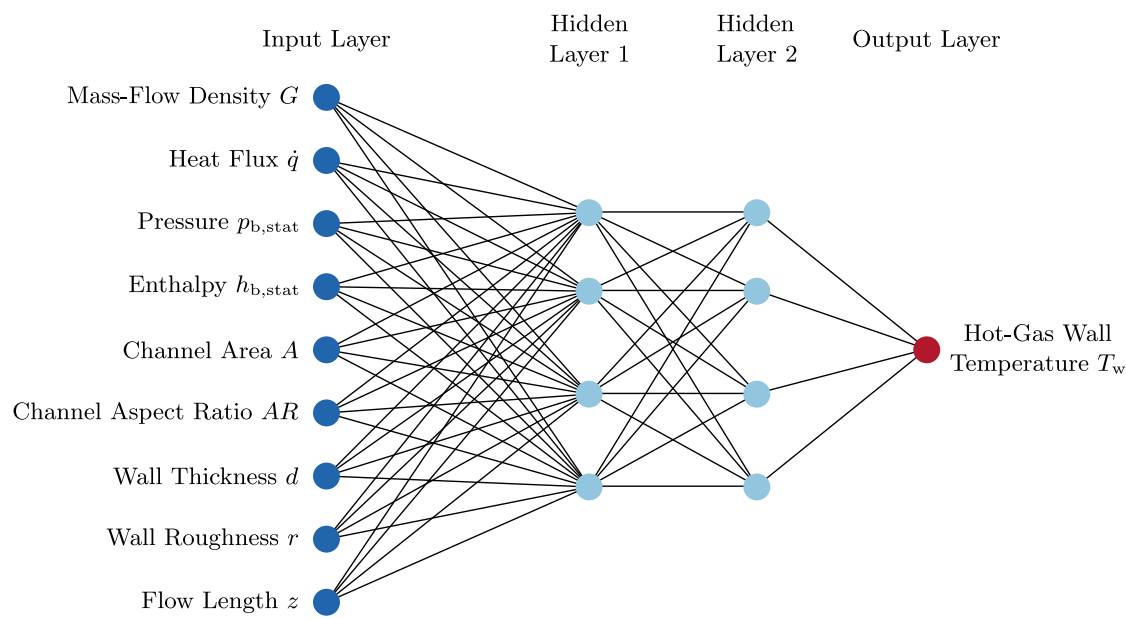

Fig. 7 Exemplary network architecture with two fully connected hidden layers.

neuron of one layer is linked with all neurons of the next layer. Figure 7 shows an exemplary model with two hidden layers, four neurons per hidden layer, and all input parameters. The optimal number of hidden layers and neurons depends on the specific problem and data, respectively. To find the best network architecture and training parameters, one needs to split the available data into suitable training and validation sets. Therefore, $90 \%$ of the data points are randomly selected for training, and the rest is held back for validation. However, under a covariate shift, data points should be weighted according to their so-called importance, which can be calculated by kernel density estimations, when calculating error measures for training and validation. For further details, the reader is referred to the work of Sugiyma et al. [39].

Given training and validation data, a classical grid search and a random search can be used to determine the optimal parameters. Bergstra and Bengio [40] showed that a random search algorithm performs as well as a grid search but with less computational cost. The proposed ANN uses ReLUs for the activation functions of the hidden neurons, and a linear unit is employed for the continuous output. During training, the weight and bias update is calculated with the ADAM optimizer, which is an extension of the classic stochastic gradient descent algorithm [41]. For faster and more robust learning, all inputs are automatically scaled and standardized with the StandardScaler from Scikit-Learn [42]. The cost function is given by a mean squared error term plus an extra term for $L 2$ regularization, as in Eq. (3). The model is generated and trained with KERAS, which is an open-source ANN library written in Python [43]. A random search of 500 different hyperparameter combinations and network architectures leads to the following optimal model: 1) four hidden layers, 2) 408 neurons per hidden layer, 3) L2 regularization with $\alpha=0.1$, 4) a minibatch size of 4096, and 5) 150 epochs.

The training takes about 15 min on a Nvidia Quadro P4000 GPU.

\section{B. Results and Visualization}

Figure 8 compares predicted and targeted values for the wall temperature. One can see that the proposed network achieves a convincing precision. The mean absolute error (MAE) of the wall temperature prediction is $8.38 \mathrm{~K}$ on the training set and $8.40 \mathrm{~K}$ on the validation set with standard deviations of 17.7 and $18.5 \mathrm{~K}$, respectively. The reason for the smaller error on the training data is the fact that the training data were directly used to optimize the model's weights, but the performance on the validation data is still impressive. One can conclude that a suitable selection of input variables is chosen to predict the maximum wall temperature with high precision. Furthermore, the amount of data samples is sufficient to train the network. Hence, one would conclude that the network has generalized well and does not overfit.

Nevertheless, there is still the risk of overfitting, especially because of the empty regions in the input space, which are also present in the validation set. To evaluate the quality of the ANN model, it is necessary to study the performance on an independent test set with yet unseen data. For this purpose, 25 further CFD calculations for five different channel geometries are made, and the resulting maximum wall temperatures are compared with the predictions of the ANN. To include various engine sizes and operation conditions, the boundary conditions are varied in a wide range, leading to lower but also higher wall temperatures. As both channel geometries and operational conditions differ from those of the training and validation data, the test set is an unbiased and independent performance measure for the ANN. As an example, the input parameters of six simulations are presented in Table 1 . The reader is referred to the Appendix for a detailed overview of the training and test data distributions.

Figure 9 shows the maximum wall temperature as a function of the axial length for both the CFD simulation and the ANN. The MAE is $16.0 \mathrm{~K}$ with a standard deviation of $12.0 \mathrm{~K}$. Overall, the ANN shows
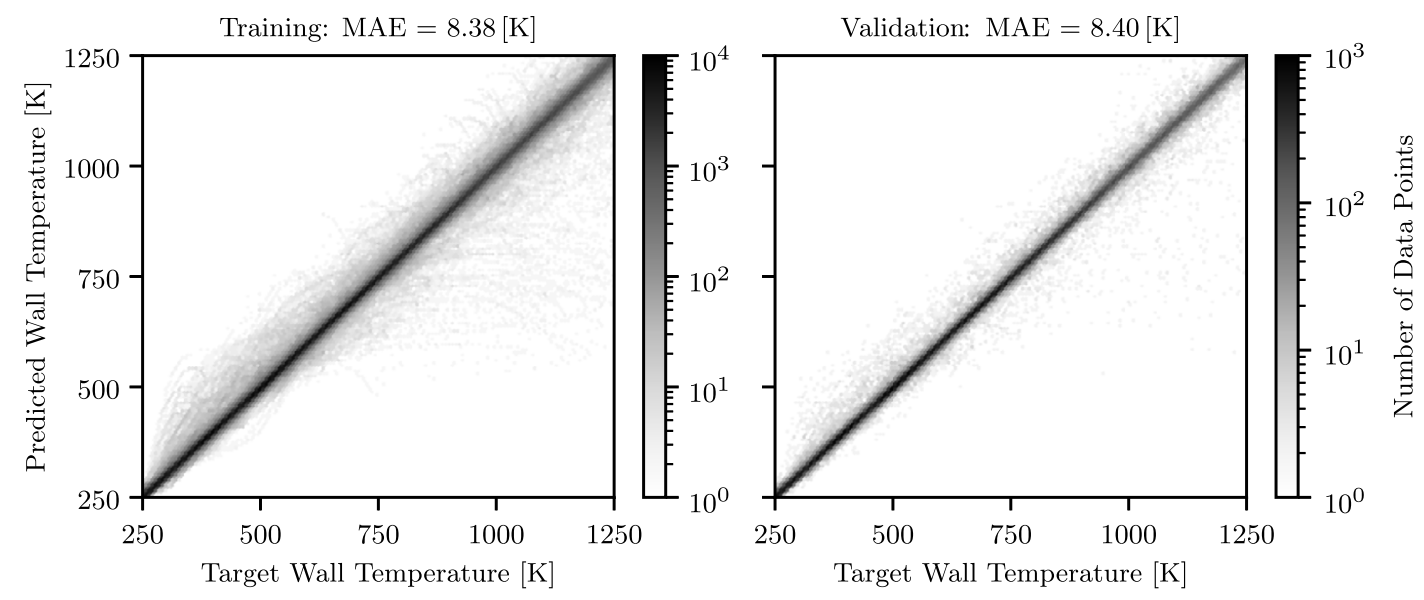

Fig. 8 Training and validation results for the proposed model. 
Table 1 Exemplary boundary conditions for the test dataset

\begin{tabular}{lcccccccr}
\hline \hline Test case, - & $T_{\text {in }}, \mathrm{K}$ & $p_{\text {out }}$, bar & $\dot{q}, \mathrm{~mW} / \mathrm{m}^{2}$ & $G, \mathrm{~kg} /\left(\mathrm{m}^{2} \cdot \mathrm{s}\right)$ & $A, \mathrm{~mm}^{2}$ & $A R,-$ & $d, \mathrm{~mm}$ & $r, \mu \mathrm{m}$ \\
\hline 1 & 140 & 80 & 49 & 11,700 & 1.9 & 2.0 & 0.83 & 2.1 \\
2 & 131 & 217 & 81 & 11,700 & 4.1 & 4.1 & 0.90 & 3.0 \\
3 & 173 & 129 & 57 & 23,900 & 7.4 & 3.7 & 1.14 & 3.0 \\
4 & 127 & 57 & 55 & 26,000 & 6.0 & 7.5 & 0.96 & 14.2 \\
5 & 290 & 51 & 14 & 10,100 & 7.4 & 3.7 & 1.14 & 1.7 \\
6 & 148 & 174 & 37 & 13,200 & 3.2 & 2.3 & 1.07 & 6.4 \\
\hline \hline
\end{tabular}

a convincing performance for all wall temperature regimes. Wall temperatures up to $1000 \mathrm{~K}$ and as low as $500 \mathrm{~K}$ are predicted with minimal error. By using the flow length, the trained network is also able to reproduce the nonlinear evolution in the streamwise direction.

Finally, the effect of heat transfer deterioration is learned, as test case

1 shows. It can be concluded that the ANN has captured the essential

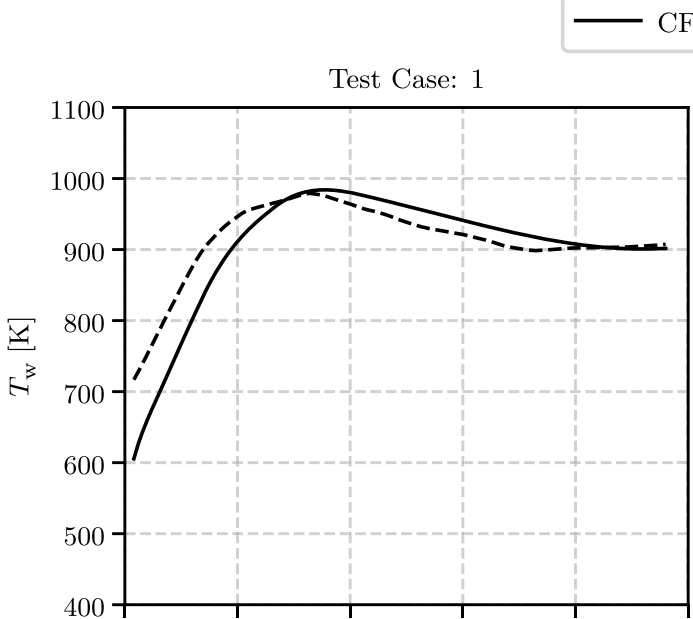

---- ANN

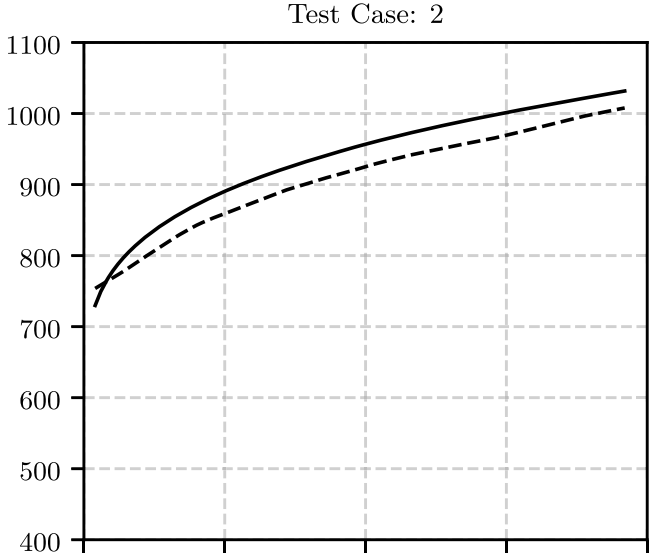

Test Case: 3

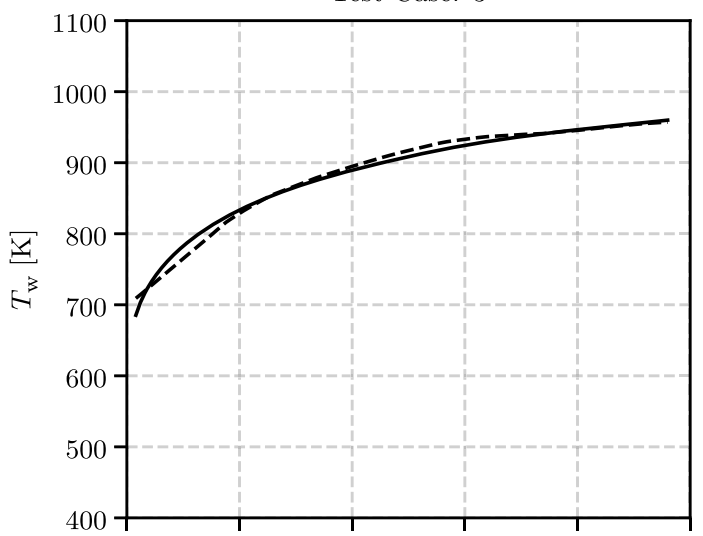

Test Case: 4

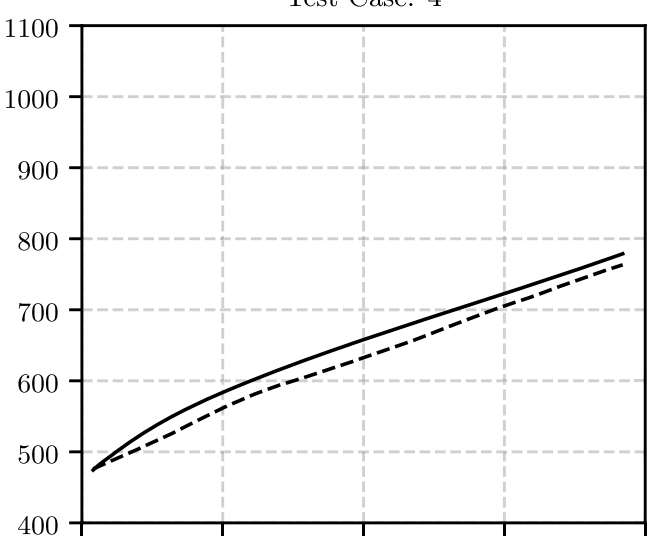

Test Case: 5

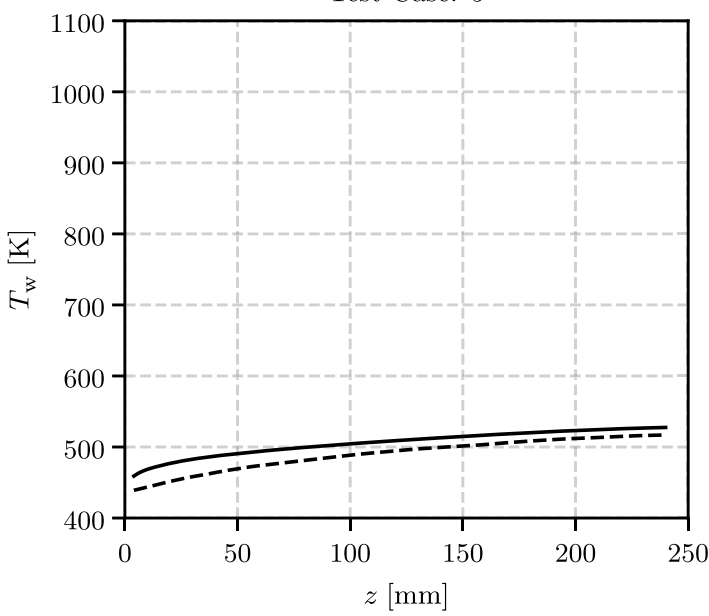

Test Case: 6

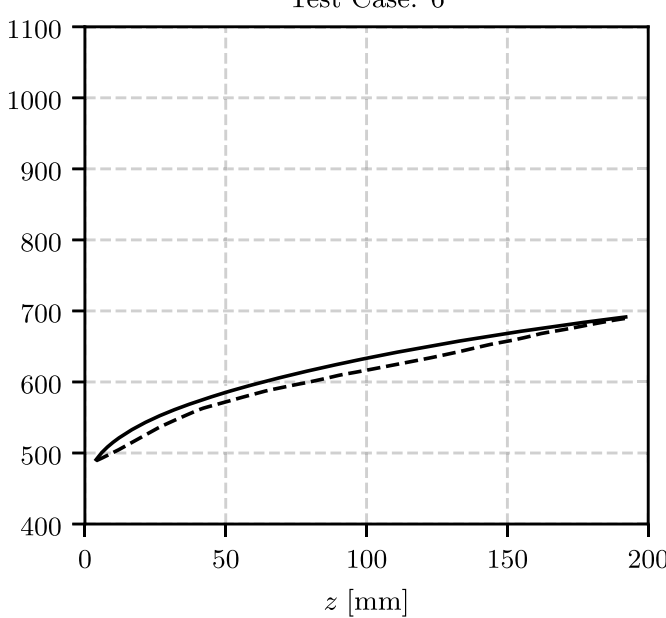

Fig. 9 Wall temperature prediction for various different design points, which are representative for different operation conditions and cooling channel geometries (see Table 1). 

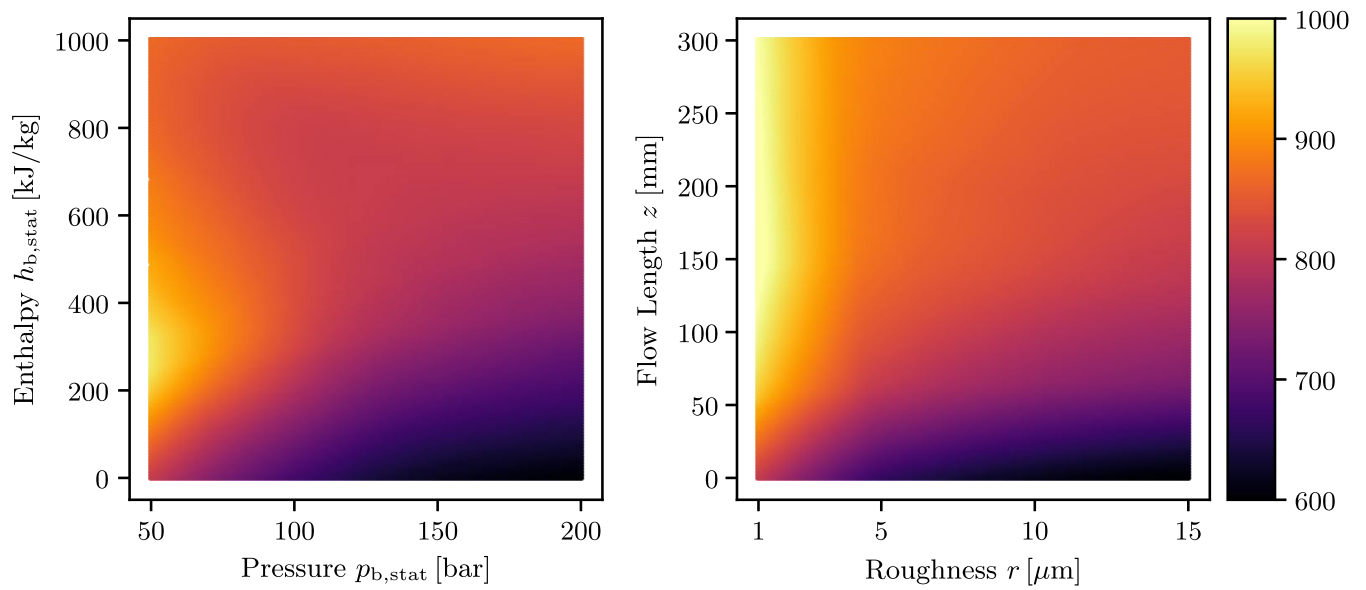

Fig. 10 Exemplary heat maps for the trained network.

underlying factors. It successfully predicts the maximum wall temperatures, even for regions in the input space where no training or validation data points are present.

Finally, it is often useful to visualize the prediction of the model for a certain range of input values. Directly observing the output helps to decide whether a model has learned the fundamental underlying factors of the given task or if it merely memorizes the training data. Additionally, it is important to visualize how the model performs in between the given input data. One can employ so-called heat maps, where two inputs are parametrically changed while all other parameters are kept the same. The output is then plotted in a two-dimensional scatter plot. Heat maps can be used to identify possible problems in terms of overfitting. For example, further investigations would be necessary if there are regions with strong unexpected discontinuities. Figure 10 illustrates the effect of varying the coolant temperature and pressure as well as the influence of the channel roughness and flow length. In terms of physical interpretation, the response seems reasonable. In general, a lower coolant bulk enthalpy or a higher bulk pressure lead to lower wall temperatures for a given heat flux because of changes in the transport properties of the coolant. Furthermore, the wall temperature builds up excessively close to the critical point. Higher roughness levels enlarge the production of turbulence in the boundary layer, thus decreasing the wall temperature. The flow lengths reflect the influence of boundary-layer growth. Finally, the wall temperature prediction changes smoothly without unphysical discontinuities.

\section{Reduced-Order Model for Cooling Channel Flow}

In addition to forecasting maximum wall temperatures, the prediction of critical variables such as pressure loss and heating of the coolant is essential for regenerative cooling design. If CFD calculations are not

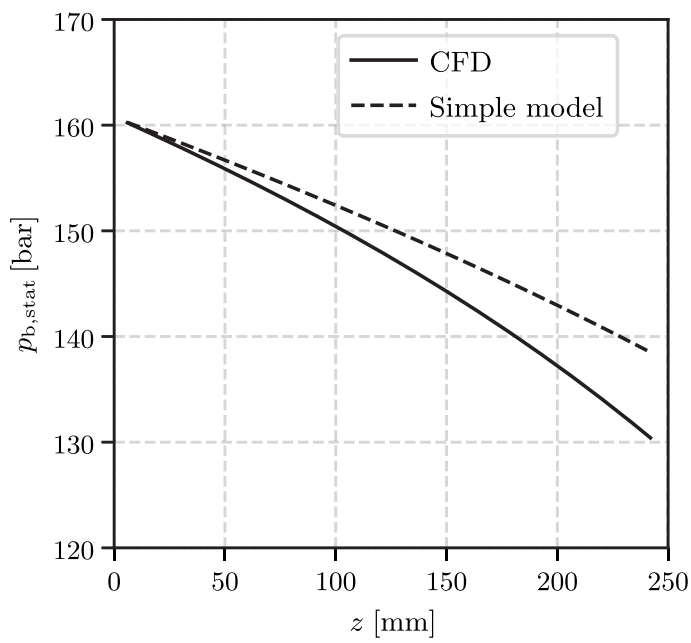

suitable due to their high computational cost, further reduced-order models are required to calculate the streamwise development of thermodynamic properties like pressure and enthalpy, whereas the ANN is used to predict the wall temperature.

\section{A. Pressure Drop Model}

The Darcy-Weisbach equation can be used to estimate the pressure loss along the cooling channels. The pressure loss in a channel segment of length $\Delta z$ is given by

$$
\Delta p=\frac{1}{2} f \rho_{b} v_{b}^{2} \frac{\Delta z}{D_{h}}
$$

where $f$ is the so-called friction factor, $\rho_{b}$ is the bulk density of the coolant, $v_{b}$ is the bulk flow velocity and $D_{h}$ is the hydraulic diameter of the channel. The friction factor $f$ can be calculated by means of a simple empirical correlation that is valid for all Reynolds numbers [44]:

$$
f=8\left[\left(\frac{8}{R e}\right)^{12}+\frac{1}{(A+B)^{1.5}}\right]^{1 / 12}
$$

with

$$
A=\left[2.457 \ln \left(\frac{1}{(7 / R e)^{0.9}+0.27\left(r / D_{h}\right)}\right)\right]^{16} \text { and } B=\left(\frac{37530}{\operatorname{Re}}\right)^{16}
$$

where $R e$ denotes the local Reynolds number, and $r$ is the surface roughness.

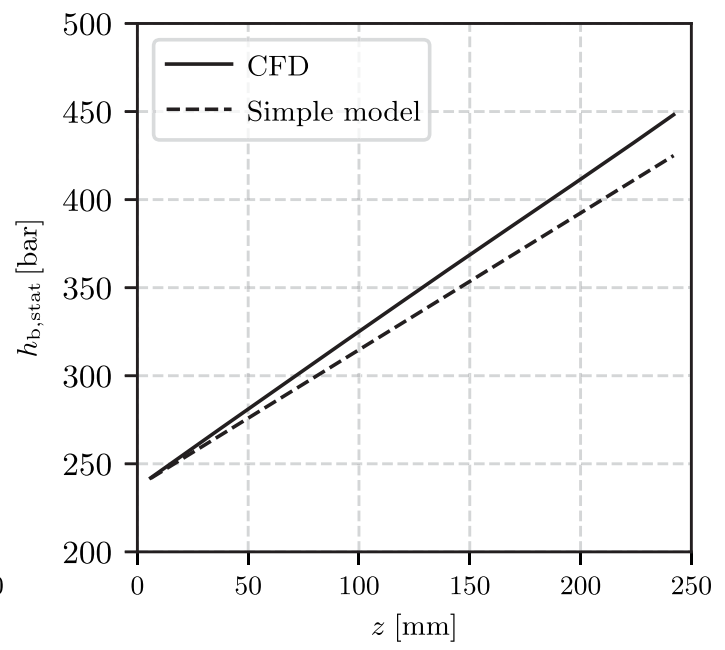

Fig. 11 Comparison between simple one-dimensional models and CFD data for bulk enthalpy and bulk pressure for an exemplary test case. 


\section{B. Enthalpy Increase Model}

The conservation of energy calculates the change of the specific total enthalpy of the fluid over a channel section of length $\Delta z$ :

$$
\begin{aligned}
h_{b, \text { tot }}(z+\Delta z) & =h_{b, \text { tot }}(z)+\frac{\dot{Q}(z, \Delta z)}{\dot{m}} \quad \text { with } \\
h_{b, \text { tot }}(z) & =h_{b, \text { stat }}(z)+\frac{1}{2} v_{b}(z)^{2}
\end{aligned}
$$

where $z$ is the streamwise coordinate, $h_{b \text {,stat }}$ is the specific bulk enthalpy of the fluid, $v_{b}$ is the bulk flow velocity, $\dot{m}$ is the mass-flow rate, and $\dot{Q}$ is the overall heat flow rate in the channel segment.

\section{Comparison with CFD}

If one adds a mass continuity equation and a suitable equation of state (or uses the NIST database), one obtains a complete reduced-order model for supercritical methane flowing in a rocket engine cooling channel. The predictions of the reduced-order model can be compared

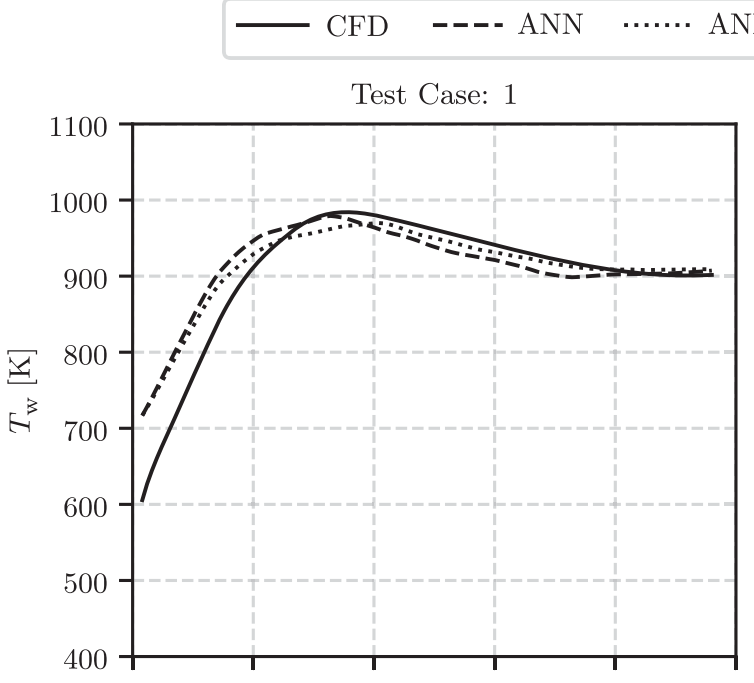

Test Case: 3

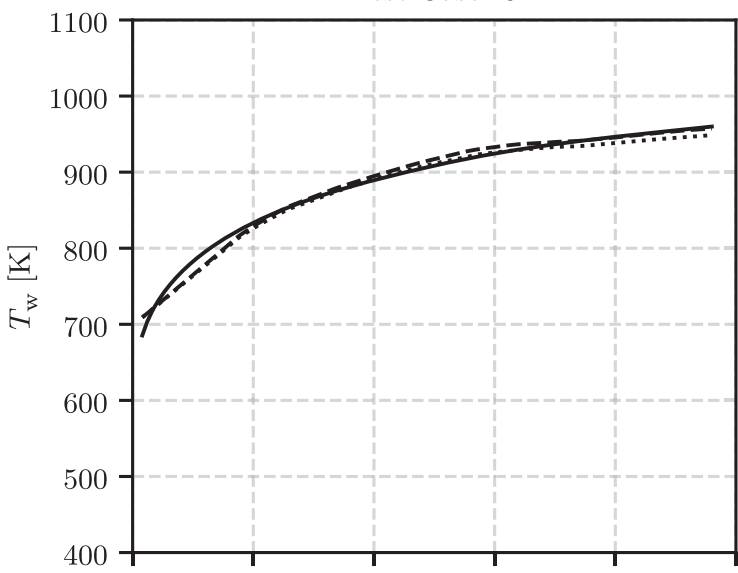

Test Case: 5

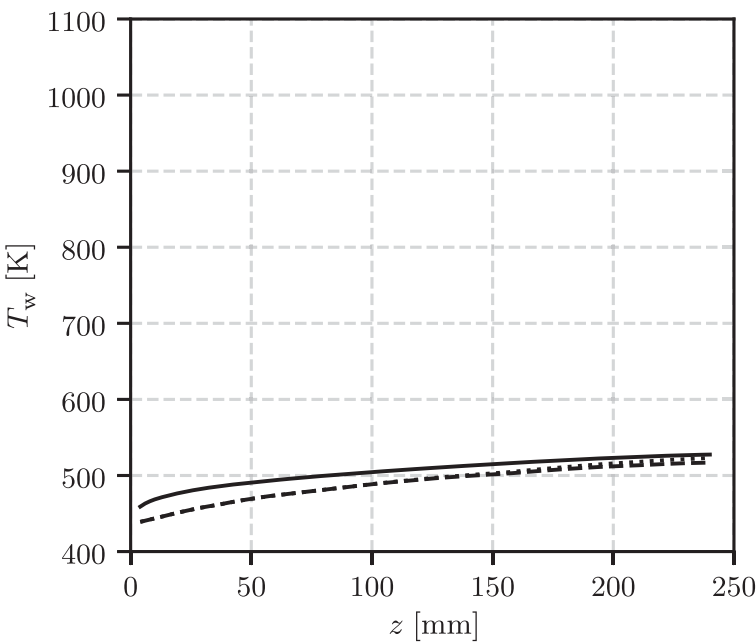

\section{+ Simple Model for Pressure and Enthalpy}

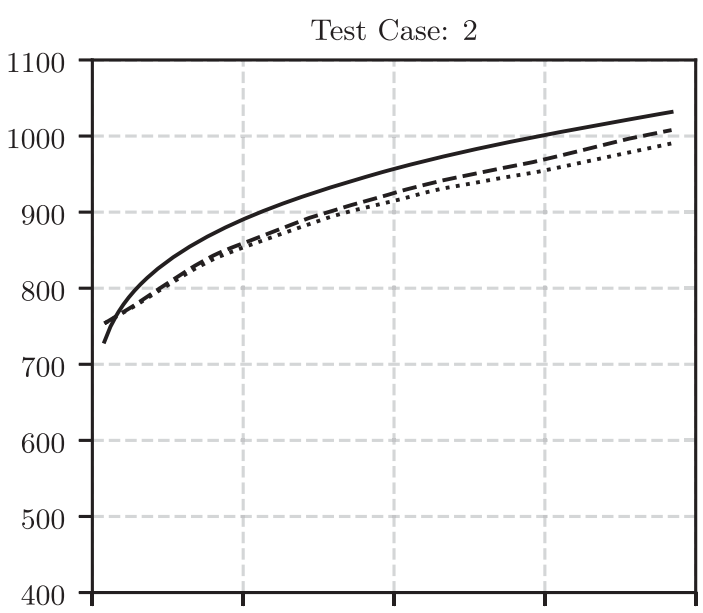

Test Case: 4

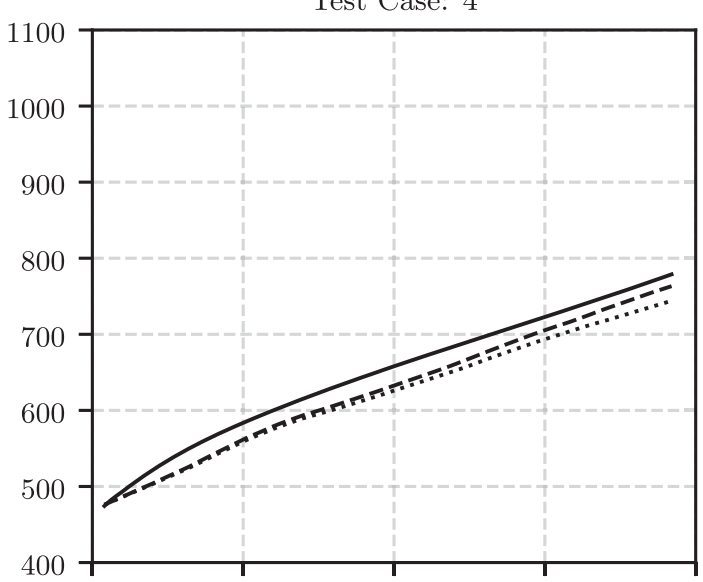

Test Case: 6

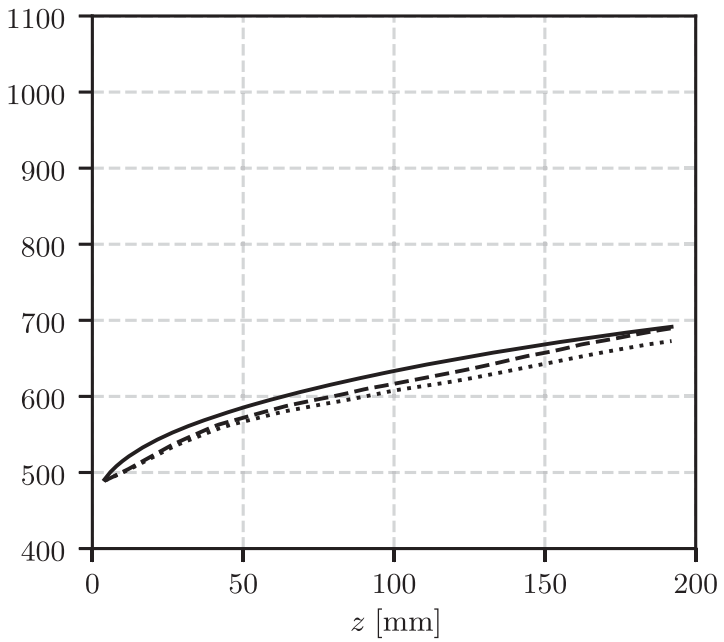

Fig. 12 Comparison of wall temperatures for CFD, the reduced-order model, and a hybrid model that uses pressure and enthalpy from CFD and the ANN for wall temperature prediction. 
with the results of a full CFD calculation. First, Fig. 11 shows the evolution of the bulk pressure and bulk enthalpy for an exemplary test case. Although error propagation increases the error in the streamwise direction, the simple models produce results with sufficient accuracy. The mean absolute percentage errors for enthalpy and pressure on the entire test dataset are 4.3 and $4.2 \%$, respectively. Thus, these models can be used to calculate pressure and enthalpy along a channel that, in turn, the ANN can use as input for the wall temperature prediction. Figure 12 shows the wall temperature prediction for the proposed network using the reduced-order models for input generation. The error only marginally increases from 16.0 to $19.6 \mathrm{~K}$ when using the reduced-order model for pressure and enthalpy calculations. In summary, the proposed reduced-order model is able to predict the evolution of the bulk pressure, the bulk enthalpy, and the resulting maximum wall temperature for low heat fluxes in the range of $10 \mathrm{MW} \cdot \mathrm{m}^{-2}$ (test case 5), medium heat fluxes (test cases 1 and 6), as well as very high heat fluxes up to $80 \mathrm{MW} \cdot \mathrm{m}^{-2}$ (test cases 2,3 , and 4 ), which can occur in the nozzle throat of a liquid rocket engine.

\section{Performance Assessment}

Although ANNs require a time-intensive training phase, the predictive speed is very high because the network is just a composite function that multiplies matrices and vectors together. Additionally, the numerical effort does not depend on the actual value of the inputs (e.g., channel area); whereas CFD simulations need increasingly more time with larger model sizes, and thus a higher number of mesh elements. For the solutions presented in this paper, the CFD calculation of one straight channel segment takes up to $1 \mathrm{~h}$, depending on the channel cross section; whereas the reduced-order model delivers the result after $0.6 \mathrm{~s}$. This comparison shows the great potential of data-driven surrogate models for design space explorations and optimization loops.

\section{Conclusions}

In this paper, an ANN was successfully trained to predict the maximum wall temperature for each cylindrical section of a rocket combustion chamber wall, given a regenerative cooling design using supercritical methane and suitable boundary conditions. The network was trained on data generated by CFD simulations of straight cooling channel segments. The ANN predicts the wall temperature for previously unseen test cases, including different channel geometries and operation conditions, with an MAE of $16.0 \mathrm{~K}$. Furthermore, the prediction of an entire channel segment takes only $0.6 \mathrm{~s}$, which is at least $10^{3}$ times faster than comparable three-dimensional CFD simulations. Thus, this numerically efficient method constitutes a convincing building block of a reduced-order model for supercritical methane flowing in rocket engine cooling channels. Also shown are which further reduced-order models can be added to obtain a suitable description for cooling channel design considerations. The presented methodology can be used to generate predictions with a precision similar to full CFD calculations and, after training, the answer only takes a fraction of the computation time of a comparable CFD simulation. Therefore, it is well suited for optimization loops and as a component of system analysis tools.

However, ANNs have disadvantages too. On the one hand, there are disadvantages that all data-driven surrogate models share. The data sample selection determines the reachable accuracy. First, if the underlying data are wrong, the resulting model will be wrong as well. For the described methodology, this means that it only works if there is a CFD code available that can model all relevant effects, e.g., heat transfer deterioration or the correct influence of different surface roughness levels. Second, depending on the complexity of the problem, the construction of a precise approximation model requires a suitably large number of CFD solutions to provide underlying data to the ANN. This data generation can get computationally very expensive. One challenge of surrogate modeling is the generation of a model that is as accurate as needed, and using as few simulation evaluations as possible. It would be interesting to study how the additional use of experimental data could improve the situation. On the other hand, there are disadvantages that are typical for ANNs. ANNs are not able to extrapolate, but they only provide reliable predictions within the region of the input space that is populated with training points. It is important to take this into account when using ANN-based models for design space exploration or optimization. Furthermore, due to the high number of parameters, these algorithms often lack a deeper understanding of the fundamental physics. Thus, domain knowledge and the understanding of physical processes are still crucially important to evaluate and justify the prediction of data-driven algorithms.

The present work can be improved in many directions. Clearly, the data generation process is not optimal. The density of the data points is too far from being uniform in the input space of interest. In future research, an optimization of the data generation should be studied. Building on this, the question should be examined of how much data are needed to reach a certain accuracy. A different choice of input parameters may increase the precision. Parameters like the boundarylayer thickness were not explicitly used in the current model. A further extension should study the consideration of curvature effects. It is well known that centrifugal forces induce recirculation phenomena in the flow that influence the heat transfer and should not be neglected. Eventually, the performance of ANNs should be compared with other types of surrogate models for the task of wall temperature prediction and heat transfer modeling, respectively. Overall, it is hoped that the current work will serve as a basis for future studies regarding the application of ANNs in the field of rocket engine design.

\section{Appendix: Data Distributions}

Tables $\mathrm{A} 1$ and $\mathrm{A} 2$ give an overview of the mean value, standard deviation, and different percentiles of the most relevant thermodynamic properties of the coolant, the channel geometries, and the resulting wall temperature at the hot-gas wall for the training and test datasets.

Table A1 Mean values, standard deviations, and percentiles of the training data

\begin{tabular}{lrrrrrrrrrrr}
\hline \hline & $T_{b}, \mathrm{~K}$ & $h_{b}, \mathrm{~kJ} / \mathrm{kg}$ & $p_{b}, \mathrm{bar}$ & $v_{b}, \mathrm{~m} / \mathrm{s}$ & $G, \mathrm{~kg} / \mathrm{sm}^{2}$ & $\dot{q}, \mathrm{MW} / \mathrm{m}^{2}$ & $r, \mu \mathrm{m}$ & $A, \mathrm{~mm}^{2}$ & $A R,-$ & $d, \mathrm{~mm}$ & $T_{w}, \mathrm{~K}$ \\
\hline Mean & 251 & 566 & 125 & 126 & 18,483 & 36 & 6.9 & 6.7 & 4.4 & 1.0 & 669 \\
Standard deviation & 84 & 317 & 42 & 78 & 8,078 & 24 & 6.1 & 3.2 & 3.1 & 0.1 & 302 \\
$1 \%$ & 123 & 56 & 53 & 18 & 3,027 & 9 & 0.2 & 1.0 & 1.0 & 0.8 & 230 \\
$25 \%$ & 183 & 279 & 90 & 64 & 12,500 & 10 & 1.0 & 5.0 & 1.7 & 1.0 & 426 \\
$50 \%$ & 240 & 572 & 119 & 109 & 17,500 & 30 & 5.0 & 5.0 & 3.5 & 1.0 & 620 \\
$75 \%$ & 302 & 790 & 158 & 174 & 25,000 & 50 & 15.0 & 10.0 & 9.2 & 1.0 & 854 \\
$99 \%$ & 433 & 1,175 & 215 & 357 & 35,000 & 80 & 20.0 & 10.0 & 9.2 & 1.2 & 1,482 \\
\hline \hline
\end{tabular}

Table A2 Mean values, standard deviations, and percentiles of the test data

\begin{tabular}{lccccccccccc}
\hline \hline & $T_{b}, \mathrm{~K}$ & $h_{b}, \mathrm{~kJ} / \mathrm{kg}$ & $p_{b}, \mathrm{bar}$ & $v_{b}, \mathrm{~m} / \mathrm{s}$ & $G, \mathrm{~kg} / \mathrm{sm}^{2}$ & $\dot{q}, \mathrm{MW} / \mathrm{m}^{2}$ & $r, \mu \mathrm{m}$ & $A, \mathrm{~mm}^{2}$ & $A R,-$ & $d, \mathrm{~mm}$ & $T_{w}, \mathrm{~K}$ \\
\hline Mean & 267 & 620 & 128 & 119 & 16402 & 42 & 5.4 & 4.5 & 3.8 & 1.0 & 741 \\
Standard deviation & 93 & 343 & 44 & 57 & 7070 & 21 & 3.9 & 2.1 & 1.9 & 0.1 & 177 \\
\hline \hline
\end{tabular}




\section{References}

[1] Sutton, G. P., History of Liquid Propellant Engines, AIAA, Reston, VA, 2005, pp. 33-53.

[2] Caisso, P., et al., "A Liquid Propulsion Panorama," Acta Astronautica, Vol. 65, Nos. 11-12, 2009, pp. 1723-1737. https://doi.org/10.1016/j.actaastro.2009.04.020

[3] Kato, T., Terakado, D., Nanri, H., Morito, T., Masuda, I., Asakawa, H., Sakaguchi, H., Ishikawa, Y., Inoue, T., Ishihara, S., et al., "Subscale Firing Test for Regenerative Cooling LOX/Methane Rocket Engine," EUCASS 2017, EUCASS, Milano, Italy, 2017. https://doi.org/10.13009/EUCASS2017-381

[4] Simontacchi, P., Blasi, R., Edeline, E., Sagnier, S., Ravier, N., Espinosa-Ramos, A., Breteau, J., and Altenhoefer, P., "PROMETHEUS: Precursor of New LowCost Rocket Engine Family," EUCASS 2019, EUCASS, Madrid, Spain, 2019.

[5] Morehead, R. L., "Project Morpheus Main Engine Development and Preliminary Flight Testing," Joint Propulsion Conference 2011, AIAA Paper 2011-5927, 2011.

[6] Deeken, J. C., Oschwald, M., and Schlechtriem, S., "LUMEN Demonstrator-Project Overview," ISTS 2019, ISTS, Fukui, Japan, 2019.

[7] Liang, K., Yang, B., and Zhang, Z., "Investigation of Heat Transfer and Coking Characteristics of Hydrocarbon Fuels," Journal of Propulsion and Power, Vol. 14, No. 5, 1998, pp. 789-796. https://doi.org/10.2514/2.5342

[8] Burkhardt, H., Sippel, M., Herbertz, A., and Klevanski, J., "Kerosene vs. Methane: A Propellant Tradeoff for Reusable Liquid Booster Stages," Journal of Spacecraft and Rockets, Vol. 41, No. 5, 2004, pp. 762-769. https://doi.org/10.2514/1.2672

[9] Pizzarelli, M., Nasuti, F., Onofri, M., Roncioni, P., Votta, R., and Battista, F., "Heat Transfer Modeling for Supercritical Methane Flowing in Rocket Engine Cooling Channels," Applied Thermal Engineering, Vol. 75, Jan. 2015, pp. 600-607.

https://doi.org/10.1016/j.applthermaleng.2014.10.008

[10] Hahn, R., Waxenegger, G., Deeken, J., Oschwald, M., and Schlechtriem, S., "Utilization of LOx/LCH4 for Expander-Bleed Cycle at Upper Stage Engine Application," EUCASS 2017, EUCASS, Milano, Italy, 2017. https://doi.org/10.13009/EUCASS2017-370

[11] Leonardi, M., Nasuti, F., and Onofri, M., "Basic Analysis of a LOX/Methane Expander Bleed Engine,” EUCASS 2017, EUCASS, Milano, Italy, 2017. https://doi.org/10.13009/EUCASS2017-332

[12] Banuti, D., "Crossing the Widom-Line-Supercritical Pseudo-Boiling," Journal of Supercritical Fluids, Vol. 98, March 2015, pp. 12-16. https://doi.org/10.1016/j.supflu.2014.12.019

[13] Simeoni, G. G., Bryk, T., Gorelli, F. A., Krisch, M., Ruocco, G., Santoro, M., and Scopigno, T., "The Widom Line as the Crossover Between Liquid-Like and Gas-Like Behaviour in Supercritical Fluids," Nature Physics, Vol. 6, No. 7, 2010, pp. 503-507. https://doi.org/10.1038/nphys1683

[14] Urbano, A., and Nasuti, F., "Parametric Analysis of Heat Transfer to Supercritical-Pressure Methane," Journal of Thermophysics and Heat Transfer, Vol. 26, No. 3, 2012, pp. 450-463. https://doi.org/10.2514/1.T3840

[15] Urbano, A., and Nasuti, F., "Onset of Heat Transfer Deterioration in Supercritical Methane Flow Channels," Journal of Thermophysics and Heat Transfer, Vol. 27, No. 2, 2013, pp. 298-308. https://doi.org/10.2514/1.T4001

[16] Locke, J., and Landrum, D., "Study of Heat Transfer Correlations for Supercritical Hydrogen in Regenerative Cooling Channels," Journal of Propulsion and Power, Vol. 24, No. 1, 2008, pp. 94-103. https://doi.org/10.2514/1.22496

[17] Dittus, F., and Boelter, L., "Heat Transfer in Automobile Radiators of the Tubular Type," International Communications in Heat and Mass Transfer, Vol. 12, No. 1, 1985, pp. 3-22. https://doi.org/10.1016/0735-1933(85)90003-X

[18] Huzel, D. K., and Huang, D. H., Modern Engineering for Design of Liquid-Propellant Rocket Engines, AIAA, Reston, VA, 1992, pp. 84-91.

[19] Waxenegger, G., Riccius, J., Zametaev, E., Deeken, J., and Sand, J., "Implications of Cycle Variants, Propellant Combinations and Operating Regimes on Fatigue Life Expectancies of Liquid Rocket Engines," EUCASS 2017, EUCASS, Milano, Italy, 2017. https://doi.org/10.13009/EUCASS2017-69

[20] Cook, R., "Methane Heat Transfer Investigation," NASA CR-171199, 1984.

[21] Pizzarelli, M., Carapellese, S., and Nasuti, F., "A Quasi-2-D Model for the Prediction of the Wall Temperature of Rocket Engine Cooling Channels," Numerical Heat Transfer, Part A: Applications, Vol. 60, No. 1, 2011, pp. 1-24. https://doi.org/10.1080/10407782.2011.578011

[22] Ruan, B., and Meng, H., "Supercritical Heat Transfer of CryogenicPropellant Methane in Rectangular Engine Cooling Channels," Journal of Thermophysics and Heat Transfer, Vol. 26, No. 2, 2012, pp. 313-321. https://doi.org/10.2514/1.T3670
[23] Pizzarelli, M., Nasuti, F., and Onofri, M., "CFD Analysis of Transcritical Methane in Rocket Engine Cooling Channels," Journal of Supercritical Fluids, Vol. 62, Feb. 2012, pp. 79-87. https://doi.org/10.1016/j.supflu.2011.10.014

[24] Wang, L., Chen, Z., and Meng, H., "Numerical Study of Conjugate Heat Transfer of Cryogenic Methane in Rectangular Engine Cooling Channels at Supercritical Pressures," Applied Thermal Engineering, Vol. 54, No. 1, 2013, pp. 237-246. https://doi.org/10.1016/j.applthermaleng.2013.02.007

[25] Negishi, H., Daimon, Y., and Kawashima, H., "Computational Analysis of Supercritical and Transcritical Flow in Cooling Channels with Rough Surface," Joint Propulsion Conference 2018, AIAA Paper 2018-4465, 2018.

[26] Haemisch, J., Suslov, D., and Oschwald, M., "Experimental Analysis of Heat Transfer Process in Cooling Channels of a Subscale Combustion Chamber at Real Thermal Conditions for Cryogenic Hydrogen and Methane," Space Propulsion Conference 2018, Seville, Spain, 2018.

[27] Haemisch, J., Suslov, D., and Oschwald, M., "Experimental Study of Methane Heat Transfer Deterioration in a Subscale Combustion Chamber," Journal of Propulsion and Power, Vol. 35, No. 4, 2019, pp. 819-826. https://doi.org/10.2514/1.B37394

[28] Hornik, K., Stinchcombe, M., and White, H., "Multilayer Feedforward Networks are Universal Approximators," Neural Networks, Vol. 2, No. 5, 1989, pp. 359-366. https://doi.org/10.1016/0893-6080(89)90020-8

[29] Sudakov, O., Koroteev, D., Belozerov, B., and Burnaev, E., "Artificial Neural Network Surrogate Modeling of Oil Reservoir: A Case Study," International Symposium on Neural Networks 2019, Moscow, Russia, 2019. https://doi.org/10.1007/978-3-030-22808-8_24

[30] Dresia, K., Waxenegger-Wilfing, G., Riccius, J., and Oschwald, M., "Numerically Efficient Fatigue Life Prediction of Rocket Combustion Chambers Using Artificial Neural Networks," EUCASS 2019, EUCASS, Madrid, Spain, 2019. https://doi.org/10.13009/EUCASS2019-264

[31] Scalabrin, G., and Piazza, L., "Analysis of Forced Convection Heat Transfer to Supercritical Carbon Dioxide Inside Tubes Using Neural Networks," International Journal of Heat and Mass Transfer, Vol. 46, No. 7, 2003, pp. 1139-1154. https://doi.org/10.1016/S0017-9310(02)00382-4

[32] Scalabrin, G., Piazza, L., and Condosta, M., "Convective Cooling of Supercritical Carbon Dioxide Inside Tubes: Heat Transfer Analysis Through Neural Networks," International Journal of Heat and Mass Transfer, Vol. 46, No. 23, 2003, pp. 4413-4425. https://doi.org/10.1016/S0017-9310(03)00256-4

[33] Chang, W., Chu, X., Fareed, A. F. B. S., Pandey, S., Luo, J., Weigand, B., and Laurien, E., "Heat Transfer Prediction of Supercritical Water with Artificial Neural Networks," Applied Thermal Engineering, Vol. 131, Feb. 2018, pp. 815-824. https://doi.org/10.1016/j.applthermaleng.2017.12.063

[34] Dresia, K., "Prediction of Heat Transfer in Methane for Liquid Rocket Engines Using Artificial Neural Networks," M.S. Thesis, RWTH Aachen Univ., Aachen, Germany, 2018.

[35] Goodfellow, I., Bengio, Y., and Courville, A., Deep Learning, MIT Press, Cambridge, MA, 2017, pp. 96-223.

[36] ANSYS CFX-Solver Theory Guide, Release 18.0, ANSYS, Inc., Canonsburg, PA, 2017, pp. 1-75.

[37] Linstrom, P., NIST Chemistry WebBook: NIST Standard Reference Database 69 (online database), National Inst. of Standards and Technology, Gaithersburg, MD, 1997. https://doi.org/10.18434/t4d303

[38] Oschwald, M., Suslov, D., and Woschnak, A., "Einfluss der Temperaturabhängigkeit der Materialeigenschaften auf den Wärmehaushalt in Regenerativ Gekühlten Brennkammern,” DGLR Jahrbuch 2004, Deutsche Gesellschaft für Luft-und Raumfahrt (DGLR), Bonn, Germany, 2004, pp. 1-8 (in-German).

[39] Sugiyama, M., Krauledat, M., and Müeller, K.-R., "Covariate Shift Adaptation by Importance Weighted Cross Validation," Journal of Machine Learning Research, Vol. 8, May 2007, pp. 985-1005.

[40] Bergstra, J., and Bengio, Y., "Random Search for Hyper-Parameter Optimization," Journal of Machine Learning Research, Vol. 13, No. 1, 2012, pp. 281-305.

[41] Kingma, D. P., and Ba, J., "Adam: A Method for Stochastic Optimization," International Conference on Learning Representations, Banff, Canada, 2014.

[42] Pedregosa, F., Varoquaux, G., Gramfort, A., Michel, V., Thirion, B., Grisel, O., Blondel, M., Prettenhofer, P., Weiss, R., Dubourg, V., et al., "Scikit-Learn: Machine Learning in Python," Journal of Machine Learning Research, Vol. 12, Oct. 2011, pp. 2825-2830.

[43] Chollet, F., et al., "Keras," 2015, https://keras.io [retrieved 27 Dec. 2019].

[44] Churchill, S., "Friction Factor Equation Spans All Fluid-Flow Regimes," Chemical Engineering Journal, Vol. 84, No. 24, 1977, pp. 91-92. 